\title{
Composição nutricional de pães do tipo bisnaguinha e comparação com a legislação de rotulagem nutricional
}

\author{
Nutritional composition of bread and comparison with the \\ legislation of nutritional labeling
}

\author{
Jéssica Schellin Tavares \\ Mayara da Cunha Mendes \\ Santiago Rullmann Passos \\ Andressa de Assis Lourenço \\ Bruna dos Anjos Pederzoli \\ Chaiane Goulart Soares
}

Fabiana Torma Botelho*

\begin{abstract}
RESUMO
O presente estudo teve como objetivo analisar a composição nutricional dos pães do tipo bisnaguinha destinados ao público infantil, comparar os resultados com a informação nutricional contida no rótulo e com a legislação vigente. Foram analisadas seis marcas de pães, em relação aos teores de proteína, gordura, fibras, carboidrato, umidade, cinzas, sódio e valor calórico, seguindo os métodos do Adolfo Lutz e da Association of Official Analytical Chemists. Os resultados encontrados foram comparados com os valores apresentados na rotulagem nutricional das embalagens e com a tolerância de 20\% preconizada pela Resolução da Diretoria Colegiada n 360 da Agência Nacional de Vigilância Sanitária, classificando os pães de acordo ou não com a legislação vigente. Das seis marcas de pães do tipo bisnaguinha analisadas, todas estavam em desacordo com a legislação vigente, pois apresentaram nutrientes em desacordo com os $20 \%$ estabelecidos pela legislação.
\end{abstract}

PALAVRAS-CHAVE: Nutrientes; Informação Nutricional; Alimentação Infantil; Vigilância Sanitária

\begin{abstract}
This study aimed to analyze the nutritional composition of bisnaguinha, a type of bread for the children's segment, and to compare the results with the nutritional information on the label and with current legislation. Six brands of breads were analyzed in relation to protein, fat, fiber, carbohydrates, moisture, ash, sodium and calories, following the methods of Adolfo Lutz and the Association of Official Analytical Chemists. The results were compared with the values in the nutritional labeling of packages and the tolerance of $20 \%$ recommended by the Brazilian Resolution number 360 of the National Health Supervision Agency, classifying the breads in agreement or not with the law. Of the six brands of bisnaguinha analyzed, all were in disagreement with the current legislation, because they presented nutrients that were not in compliance with the $20 \%$ established by the legislation.
\end{abstract}

KEYWORDS: Nutrients; Nutritional Information; Food for Children; Sanitary Surveillance
Universidade Federal de Pelotas (UFPel), Pelotas, RS, Brasil

\footnotetext{
* E-mail: fabibotelho@hotmail.com
} 


\section{INTRODUÇ̃̃O}

0 rótulo identifica o alimento, tendo em vista que a rotulagem é toda inscrição, legenda, imagem ou toda matéria descritiva ou gráfica que esteja impressa sobre a embalagem do alimento ${ }^{1} \mathrm{e}$ se aplica a todo alimento que seja produzido, comercializado e embalado na ausência do consumidor ${ }^{2}$. A rotulagem nutricional é tratada nas Resoluções da Diretoria Colegiada (RDC) $n^{\circ} 259^{1}$ e $n^{\circ} 360^{2}$, da Agência Nacional de Vigilância Sanitária (Anvisa), com o objetivo de informar ao consumidor sobre o conteúdo nutricional dos alimentos embalados e comercializados, e tem como base a Lei $n^{\circ} 6.437 / 1977^{3}$, cuja infração ocasiona multa e demais sanções.

A informação nutricional contida nos rótulos dos alimentos serve como instrumento de saúde pública e tem função de garantir a saúde da população, auxiliando nas suas escolhas alimentares ${ }^{4,5}$. A rotulagem nutricional que informa corretamente as quantidades e os nutrientes que realmente estão presentes no alimento é um direito do consumidor, visto que, segundo a Lei $\mathrm{n}^{\circ} 8.078 / 1990^{6}$, os produtos alimentícios devem obter correta especificação de quantidade, características, composição e qualidade.

Foi observado em alguns estudos que os consumidores leem os rótulos dos alimentos, buscam as informações nutricionais contidas nas embalagens dos produtos alimentícios relacionadas ao valor calórico, à composição nutricional e ao prazo de validade $4,5,7,8,9$. Porém, outros estudos observaram que as informações nutricionais contidas nos rótulos não são fidedignas devido às inconformidades encontradas com relação à quantidade de nutrientes analisados, tais como carboidrato, proteína, gordura, fibras e sódio ${ }^{10,11,12,13}$.

Os pães do tipo bisnaguinha são destinados ao público infantil, visto que apresentam apelo com personagens infantis nas embalagens dos produtos. As crianças influenciam $92 \%$ das compras de produtos alimentícios, tendo como fatores determinantes para a escolha a publicidade na televisão e a presença de personagens nas embalagens ${ }^{14}$. Estudo que avaliou alimentos e bebidas industrializados mais consumidos pelo público infantil identificou 20 tipos diferentes de estratégia de marketing entre as crianças ${ }^{15}$.

Ademais, os pais podem fazer as escolhas alimentares para os lanches e merendas de seus filhos baseados nas informações nutricionais contidas nos rótulos desses alimentos ${ }^{7}$, entretanto, eles devem estar cientes sobre o tipo e a qualidade do nutriente/alimento que estão escolhendo para seus filhos. Torna-se importante o cuidado com a alimentação infantil, visto que a prevalência de excesso de peso em crianças, nos anos de 2008 e 2009, foi de 33,5\% entre as crianças de cinco a nove anos, segundo a Pesquisa de Orçamento Familiar (POF) ${ }^{16}$.

Sendo assim, o objetivo do presente estudo foi analisar, por meio de análises laboratoriais, a composição nutricional dos pães do tipo bisnaguinha destinados ao público infantil, comparar os resultados com as informações nutricionais apresentadas nos rótulos das embalagens e verificar a adequação perante a RDC $\mathrm{n}^{\circ} 360$ da Anvisa².

\section{MÉTODO}

Foram visitados os cinco maiores supermercados da cidade de Pelotas/RS e identificadas seis marcas, de diferentes empresas, de pães do tipo bisnaguinha com apelo infantil na embalagem. Tais marcas foram nomeadas de A, B, C, D, E e F.

Para as análises laboratoriais, foram adquiridos três lotes diferentes (triplicata) para cada uma das seis marcas de pão, em diferentes momentos de compra. A análise de cada nutriente foi realizada em duplicata para cada um dos três lotes, obtendo seis resultados de cada nutriente para cada uma das marcas, totalizando 36 amostras analisadas. Todas as análises foram realizadas no Laboratório de Bromatologia da Faculdade de Nutrição da Universidade Federal de Pelotas/UFPel, no período entre novembro de 2014 a maio de 2015.

Para o preparo das amostras, todo o conteúdo da embalagem foi triturado em liquidificador (marca Mondial ${ }^{\oplus}$ ) para homogeneização adequada. Após, as amostras foram colocadas em sacos plásticos hermeticamente fechados, identificados e procedeu-se em seguida às análises laboratoriais.

$\mathrm{O}$ teor de umidade foi realizado por secagem em estufa a $105^{\circ} \mathrm{C}$ até peso constante ( 16 a 24 horas) e o teor de cinzas por calcinação em mufla à temperatura de $550^{\circ} \mathrm{C}$ a $600^{\circ} \mathrm{C}$, segundo as normas do Instituto Adolfo Lutz ${ }^{17}$. A análise do teor de proteína foi realizada por meio do método Kjeldahl com modificações, no qual a amostra foi digerida até o aparecimento de coloração azul esmeralda e depois realizou-se a destilação e o processo de titulação, seguindo o método 991.20 da Association of Official Analytical Chemists $(\mathrm{AOAC})^{18}$. Para determinação de gorduras totais, foi utilizado o método Soxhlet, seguindo as normas da $\mathrm{AOAC}^{18} \mathrm{e}$, para fibras, utilizou-se o método gravimétrico, seguindo a descrição do método 044/IV nas normas analíticas do Instituto Adolfo Lutz ${ }^{17}$.

O teor de carboidrato foi calculado subtraindo de 100 os teores encontrados de proteínas, cinzas, gordura, fibras e umidade de acordo com as normas do Instituto Adolfo Lutz ${ }^{17}$. $\mathrm{O}$ valor calórico foi determinado a partir da soma do produto da multiplicação dos teores de proteína por $4 \mathrm{kcal} / \mathrm{g}$, gordura por $9 \mathrm{kcal} / \mathrm{g}$ e carboidrato por $4 \mathrm{kcal} / \mathrm{g}$, segundo a RDC $\mathrm{n}^{\circ} 360$ da Anvisa ${ }^{2}$. A análise de sódio, por determinação de cloretos de sódio $(\mathrm{NaCl})$, foi realizada por calcinação seguida de titulação com nitrato de prata, de acordo com metodologia do Instituto Adolfo Lutz ${ }^{17}$.

Todos os resultados obtidos a partir das análises da composição nutricional realizadas foram comparados com os valores apresentados na informação nutricional nos rótulos das embalagens e verificados segundo a tolerância estabelecida pela RDC $n^{\circ} 360^{2}$, classificando os pães que ultrapassaram os valores de $20 \%$ para mais ou para menos, como em desacordo com a legislação vigente. 


\section{RESULTADOS}

Das seis marcas de pães do tipo bisnaguinha analisadas, todas apresentaram algum tipo de irregularidade em relação aos nutrientes que foram analisados, com valores abaixo de $20 \%$, ultrapassando a margem imposta pela RDC $n^{\circ} 360^{2}$. Os resultados das análises e comparações com os rótulos dos alimentos encontram-se na Tabela.

O conteúdo de gordura, fibras e sódio obteve $100 \%$ de inconformidade com a legislação em todas as seis marcas de pães do tipo bisnaguinha. Salienta-se que os valores foram menores do que os apresentados nos rótulos, variando entre $28,66 \%$ a $96,38 \%$ a menos de gordura, de $71,07 \%$ a $91,50 \%$ a menos de fibras e de $37,07 \%$ a $78,67 \%$ a menos de sódio do que os valores apresentados na rotulagem nutricional impressa nas embalagens.

Já em relação ao conteúdo de proteína, três marcas estavam em desacordo com a legislação, com valores variando entre menos $21,18 \%$ a $53,13 \%$. O único nutriente dos pães do tipo bisnaguinha que não apresentou irregularidade em relação à legislação, foi o conteúdo de carboidratos, pois todas as amostras estavam de acordo com a legislação vigente.

Entretanto, ao analisar o valor calórico, apenas uma marca apresentou irregularidade perante a legislação, com 25,06\% abaixo do valor impresso na rotulagem nutricional. Essa mesma marca de pão também mostrou-se em desacordo com a legislação em relação aos nutrientes: gordura (menos 96,38\%), fibras (menos $83,25 \%$ ) e sódio (menos $78,67 \%$ ).
Importante salientar que, das seis marcas de pães analisadas, três estavam em desacordo com a legislação em relação a três nutrientes apresentados, enquanto que as outras três marcas de pães apresentaram quatro nutrientes que excederam a tolerância de $20 \%$, classificadas também em desacordo perante a legislação. Além disso, todas as irregularidades foram para valores menores do que $20 \%$.

\section{DISCUSSÃO}

Outros estudos ${ }^{10,12}$ também encontraram irregularidades em $100 \%$ das amostras de alimentos analisados e comparados com a rotulagem nutricional nas embalagens e com a legislação, assim como o presente estudo. Tais estudos analisaram a composição centesimal de batatas-palha, salgadinhos de milho e trigo, batata frita, amendoim e biscoito.

Os resultados de desacordo em relação ao conteúdo de gordura do presente estudo corroboram com outros estudos que também analisaram o conteúdo de gordura em salgadinhos de milho e trigo, batata frita, amendoim, biscoito ${ }^{12}$ e pães de queijo ${ }^{19}$, nos quais foram encontrados valores superiores ou inferiores a $20 \%$ dos valores permitidos pela $\operatorname{RDC} n^{\circ} 360^{2}$. Ademais, outro estudo que avaliou os teores de gordura total, saturada, monoinsaturada, poli-insaturada e trans de empanadas comercializadas em cantinas escolares, mostrou que, das nove amostras analisadas, todas apresentavam alguma não conformidade (extrapolando os $20 \%$ de tolerância) em, pelo menos, uma das gorduras avaliadas, principalmente em relação a gorduras trans, sendo a gordura vegetal, fonte de gorduras trans, a mais citada na lista dos ingredientes dessas empanadas ${ }^{20}$.

Tabela. Comparação entre os valores encontrados nas análises (100g) com os valores apresentados nos rótulos (100g) e adequação com a RDC n 360, com tolerância de $20 \%$.

\begin{tabular}{|c|c|c|c|c|c|c|c|}
\hline Marca & Adequação & Gordura (g) & Proteína (g) & Fibras (g) & Carboidrato (g) & Sódio (mg) & $\begin{array}{l}\text { Valor calórico } \\
\text { (kcal) }\end{array}$ \\
\hline \multirow{3}{*}{$A$} & Média análise & 2,39 & 10,64 & 0,64 & 60,81 & 107,95 & 307,31 \\
\hline & Rótulo & 4,5 & 12,25 & 3,5 & 52,5 & 407,5 & 305 \\
\hline & Adequação & $-46,88$ & $-13,14$ & $-81,6$ & 15,82 & $-73,5$ & 0,75 \\
\hline \multirow{3}{*}{ B } & Média análise & 2,5 & 8,67 & 0,21 & 63,4 & 174,04 & 310,78 \\
\hline & Rótulo & 4,75 & 11 & 2,25 & 55 & 407,5 & 310 \\
\hline & Adequação & $-47,36$ & $-21,18$ & $-90,7$ & 15,27 & $-57,29$ & 0,25 \\
\hline \multirow{3}{*}{ C } & Média análise & 2,68 & 7,49 & 0,2 & 63,55 & 231,89 & 308,28 \\
\hline & Rótulo & 4,75 & 11 & 2,25 & 55 & 407,5 & 310 \\
\hline & Adequação & $-43,57$ & $-31,9$ & $-91,1$ & 15,54 & $-43,09$ & $-0,55$ \\
\hline \multirow{3}{*}{ D } & Média análise & 1,5 & 8,96 & 0,81 & 54,71 & 201,35 & 268,18 \\
\hline & Rótulo & 5,6 & 8,4 & 2,8 & 54 & 320 & 304 \\
\hline & Adequação & $-73,21$ & 6,66 & $-71,1$ & 1,31 & $-37,07$ & $-11,78$ \\
\hline \multirow{3}{*}{$E$} & Média análise & 0,19 & 8,02 & 0,34 & 50,1 & 113,55 & 234,17 \\
\hline & Rótulo & 5,25 & 8,5 & 2 & 57,5 & 532,5 & 312,5 \\
\hline & Adequação & $-96,38$ & $-5,64$ & $-83,3$ & $-12,87$ & $-78,67$ & $-25,06$ \\
\hline \multirow{3}{*}{$\mathrm{F}$} & Média análise & 4,28 & 7,5 & 0,34 & 57,85 & 89,88 & 299,92 \\
\hline & Rótulo & 6 & 16 & 4 & 68 & 400 & 340 \\
\hline & Adequação & $-28,66$ & $-53,13$ & $-91,5$ & $-14,92$ & $-77,53$ & $-11,78$ \\
\hline
\end{tabular}

*Valores em negrito ultrapassaram a margem de $20 \%$ de tolerância estipulada pela RDC n $360^{2}$. 
Mesmo que, no presente estudo, os valores encontrados para gordura tenham sido menores do que os apresentados nos rótulos, isso não é favorável para o consumidor, pois a falta de fidedignidade nas informações disponibilizadas pode causar uma menor confiabilidade perante o produto e a empresa, ainda mais tratando-se de um alimento destinado ao público infantil. As informações contidas nos rótulos deveriam ser as mais confiáveis e fidedignas possíveis, visto que se trata de um macronutriente, cuja ingestão por crianças pode, em alguns casos, ser controlada.

Relacionando os valores encontrados de fibras com outro estudo $^{21}$, que utilizou o mesmo método de análise de fibras do presente estudo, verificou-se também valores inferiores aos que estavam apresentados nos rótulos em três marcas de barras de cereais, ultrapassando a tolerância de $20 \%$ estabelecida pela legislação. Mas tal fato pode ocorrer pelos diferentes métodos adotados, pois o método utilizado para analisar fibras em ambos os estudos pode ocasionar perdas de fibras no decorrer do processo. Entretanto, esse método também já foi adotado em diferentes estudos sobre o mesmo tema de pesquisa ${ }^{13,21,22}$.

O método mais indicado para análise de fibra alimentar (solúvel e insolúvel) seria o método enzimático, com a utilização de enzimas que simulam o meio ideal de digestão no organismo, preservando as fibras solúveis e insolúveis, porém, o custo dessas enzimas é alto, limitando muitas vezes, sua utilização, como no caso do presente estudo. 0 que ocorre na utilização do método gravimétrico é uma redução nos valores de fibras encontrados, assim como uma superestimação do valor de carboidratos, quando ele é calculado por diferença da fração "NIFEXT", sendo ideal as determinações de açúcares redutores e totais pelo método de Lane Eynon.

Embora essas determinações sejam uma limitação no presente estudo, pode-se observar que o conteúdo de carboidratos foi o único nutriente que estava conforme em todas as marcas de pães do tipo bisnaguinha analisadas, não apresentando irregularidade entre a análise por diferença e o valor apresentado no rótulo. Além disso, apenas uma marca apresentou irregularidade no valor calórico declarado, mas foi uma amostra na qual a gordura obteve redução de $96,38 \%$, o que pode ter influenciado diretamente no cálculo.

Em relação ao conteúdo de sódio, os valores encontrados em desacordo foram menores do que os apresentados nos rótulos. Já em outro estudo, o conteúdo de sódio em batatas-patha foi $51 \%$ acima do permitido pela legislação ${ }^{10}$. O sódio é um nutriente que deve ser controlado na alimentação, pois o seu excesso pode causar várias complicações à saúde, principalmente em crianças, devido aos riscos de aumento da pressão $\operatorname{arterial}^{23,24,25}$.

De acordo com a POF (2008-2009) ${ }^{16}$, o consumo médio de sódio no Brasil por pessoa é de $4.700 \mathrm{mg}$ de sódio ( $12 \mathrm{~g}$ de sal) por dia. 0 Guia Alimentar para a População Brasileira ${ }^{26}$ traz a recomendação de $5 \mathrm{~g}$ de sal por dia, portanto, as informações sobre a quantidade de sódio apresentadas na rotulagem nutricional devem ser importantes para assim auxiliar na escolha dos alimentos com menor conteúdo de sódio.

Por esse motivo, o Ministério da Saúde estabeleceu o Termo de Compromisso $\mathrm{n}^{\circ}$ 004/201127 com as indústrias de alimentos: Associação Brasileira das Indústrias de Alimentação (ABIA), Associação Brasileira das Indústrias de Massas Alimentícias (Abima), Associação Brasileira da Indústria de Trigo (Abitrigo) e Associação Brasileira da Indústria de Panificação e Confeitaria (ABIP), dentro do Plano Nacional da Redução do Sódio em Alimentos Processados, com o objetivo de reduzir a ingestão de sódio em pães (grupo que abrange os pães do tipo bisnaguinha), caldos e temperos, laticínios, biscoitos, margarina vegetal, embutidos, massas semiprontas, bolos, maionese, snacks, derivados de cereais, refeições prontas, entre outros ${ }^{28,29}$.

Dentro do Termo de Compromisso $n^{\circ} 004 / 2011^{27}$, pães do tipo bisnaguinha devem ter no máximo $430 \mathrm{mg} / 100 \mathrm{~g}$ de sódio após o ano de 2014. Analisando o conteúdo de sódio encontrado no presente estudo, todas as marcas já estão de acordo com o Termo de Compromisso, o que poderia estar relacionado à obediência ao acordo e às recomendações de redução de sódio, sendo que a rotulagem nutricional impressa no rótulo poderia não estar atualizada em relação ao novo conteúdo de sódio. Assim como no estudo de Dias, Moraes e Camara $^{30}$, no qual as quatro marcas de pães do tipo bisnaguinha analisadas também estavam abaixo da meta estabelecida para sódio. Entretanto, os autores ${ }^{30}$ chamam a atenção que a quantidade de $100 \mathrm{~g}$ de bisnaguinha (seis unidades) possui uma participação na ingestão diária de sódio que varia de $30,29 \%$ a $43,08 \%$ para a faixa etária de crianças de um a três anos e de $25,24 \%$ a $35,90 \%$ para a faixa etária de quatro a oito anos. Ressaltando ainda que os pães do tipo bisnaguinha são consumidos com alimentos com elevada concentração de sódio, tais como requeijão, queijo, manteiga, entre outros, podendo ultrapassar a recomendação diária desse nutriente.

Ademais, um outro estudo que monitorou o teor de sódio em alimentos industrializados, comparando-o com as metas de redução de sódio estipuladas por acordos voluntários do governo brasileiro com a indústria desde 2009, mostrou que, mesmo antes do prazo, sete categorias de alimentos já tinham reduzido a quantidade de sódio abaixo das metas, porém, salienta-se a falta de padronização e fragilidade no monitoramento do teor de sódio, o que pode comprometer a efetividade da estratégia que contribui com a saúde da população ${ }^{31}$. Tais dados mostram que o fortalecimento do monitoramento laboratorial de alimentos deve ser incentivado e que novos acordos são necessários para reduzir gradativamente o teor de sódio e outros nutrientes que, em excesso, podem prejudicar a saúde da população. Além disso, no presente estudo, a maior e menor concentração encontrada de sódio foi de $231,89 \mathrm{mg}$ e $89,88 \mathrm{mg}$, respectivamente, mostrando uma diferença de $142,01 \%$ de sódio entre as amostras e a possibilidade de maiores reduções desse nutriente.

No presente estudo, os teores de nutrientes analisados que mais demonstraram inadequação perante o conteúdo apresentado nos rótulos foram: gordura, fibra e sódio. Fato semelhante ocorreu no estudo de Lobanco et al. ${ }^{12}$, no qual nenhuma 
amostra dos produtos salgados estava em conformidade quanto ao conteúdo de fibra alimentar, sódio e gorduras. Nele, os métodos para gordura e sódio foram os mesmos do presente estudo, sendo apenas a análise de fibras realizada pelo método gravimétrico. Os salgadinhos de milho foram os alimentos com maior frequência de inadequação, com valores de $69 \%$ de fibra alimentar, $72 \%$ de sódio, $85 \%$ de gorduras totais e $41 \%$ de gorduras saturadas.

Referente ao conteúdo de proteína, também foi observado no estudo de Sauerbronn ${ }^{32}$ que $50 \%$ de suas amostras de massas estavam em desacordo com a legislação, com valores abaixo do rotulado, assim como no presente estudo. Os valores de proteína no estudo de Pereira et al. ${ }^{19} \mathrm{e}$ Jesus et al. ${ }^{33}$ foram menores do que os valores apresentados nos rótulos das embalagens quando analisaram pão de queijo, mostrando também inadequação. Por outro lado, no estudo de Pederzoli et al. ${ }^{22}$, que analisou bolos destinados ao público infantil, nenhuma amostra estava inadequada em relação ao conteúdo de proteína.

O conteúdo de carboidratos foi o único nutriente que estava conforme em todas as marcas no presente estudo, porém, comparando com o estudo de Fregonesi et al. ${ }^{34}$ que analisou 10 amostras de polpas de açaí, as quantidades de carboidratos $(96,67 \%)$, gorduras $(83,33 \%)$, proteínas $(70 \%)$, fibras $(83,33 \%)$ e valor calórico $(86,67 \%)$ estavam em desacordo com a $\mathrm{RDC} \mathrm{n}^{\circ} 360$, quando a comparação foi feita entre os valores encontrados nas análises $\mathrm{e}$ nos valores apresentados nos rótulos.

De acordo com Silva e Dutra ${ }^{13}$, que avaliaram a conformidade da rotulagem nutricional de preparados instantâneos do tipo "sopão", utilizando os mesmos métodos do presente estudo, todas as 21 amostras estavam de acordo com a legislação quanto ao valor calórico, mesmo que outros macronutrientes tenham apresentado irregularidades. Já o estudo de Pereira et al. ${ }^{19}$, em análises de pães de queijo, todas as três amostras estavam inadequadas perante o valor calórico, sendo que uma apresentou valor abaixo do apresentado no rótulo e as outras duas apresentaram valores acima daqueles rotulados. Enquanto que, no presente estudo, apenas uma marca estava inadequada perante a quantidade do valor calórico.

Mesmo que os pães tenham apresentado irregularidades em relação ao conteúdo de gordura em seis marcas e de proteína em três marcas, o valor calórico total não sofreu alteração em cinco amostras. A única amostra que apresentou irregularidade em relação ao valor calórico foi a que apresentou maior valor de irregularidade no conteúdo de gordura (menos $96,38 \%$ ), sendo que essa quantidade a menos pode ter influenciado na redução do valor calórico, pois a gordura representa $9 \mathrm{Kcal}$ por grama.

O valor calórico é a informação mais visualizada no rótulo pelos consumidores ${ }^{5}$, visto que os consumidores se baseiam nessas informações para fazer suas escolhas alimentares ${ }^{7} \mathrm{e}$, assim, proceder à compra dos alimentos. Embora, o valor calórico seja importante, não deve ser a única informação que determine a compra dos alimentos, pois deve-se analisar também outros nutrientes, tais como fibra, gordura, carboidrato, proteína e sódio, com funções importantes para a saúde e assim decidir sua compra baseada na qualidade nutricional dos alimentos oferecidos.

A falta de tempo para se alimentar e preparar suas refeições são dificuldades enfrentadas pelas famílias na correria do cotidiano, dificultando, muitas vezes, a prática de uma alimentação saudável. Os lanches práticos e instantâneos, fáceis de adquirir e consumir são algumas das opções escolhidas pelos pais como lanches para seus filhos. Os pães do tipo bisnaguinha são uma dessas opções, sugerido ao público infantil por conter apelos infantis em suas embalagens. Os pais ou responsáveis podem escolher os alimentos adquiridos para seus filhos com base nas informações nutricionais contidas nos rótulos e na informação nutricional ${ }^{22}$.

Mas, para que ocorra uma escolha alimentar adequada, as informações nutricionais apresentadas nos rótulos devem condizer com o produto alimentício oferecido, o que não foi observado no presente estudo com as marcas de pães analisadas, visto que todas as marcas apresentaram inconformidades perante a legislação. Portanto, o rótulo que apresenta inconformidade fere os direitos do consumidor, de acordo com o Decreto $n^{\circ} 5.903 / 2006^{35}$, que avalia práticas infracionais que atentam contra o direito básico do consumidor em obter informação adequada e clara sobre produtos e serviços adquiridos.

A informação contida no produto alimentício deve apresentar-se com clareza e exatidão para que a população tenha direito a uma informação nutricional verdadeira. Isso vale tanto para as empresas que devem buscar sempre a atualização das informações oferecidas, como para os órgãos de fiscalização e legislação, Estes órgãos permitem a utilização de tabelas de composição de alimentos prontas, propiciando erros nas informações vinculadas, visto que nem todas as tabelas apresentam metodologias e amostragem adequadas, reproduzindo erros quando as tabelas são consultadas para a elaboração da rotulagem nutricional. Ademais, informações disponibilizadas de maneira clara e exata, aumentam a credibilidade da empresa perante o consumidor e permitem melhor avaliação do consumidor em relação ao produto atender suas necessidades e ter confiança em realizar suas escolhas alimentares?.

\section{CONCLUSÃO}

Das seis amostras de pães do tipo bisnaguinha analisadas, nenhuma estava de acordo com a legislação vigente em todos os nutrientes analisados, pois apresentaram nutrientes em desacordo com os $20 \%$ estabelecidos pela legislação. Ressalta-se o importante papel tanto da indústria de alimentos em oferecer informações verdadeiras dos seus produtos aos seus clientes, como dos órgãos de fiscalização, que permitem a utilização de tabelas de composição dos alimentos que apresentam erros pelas diferenças de metodologias utilizadas e falta de padronização. Para que seja possível garantir a segurança alimentar do consumidor e fazer cumprir a legislação brasileira de rotulagem nutricional de forma fidedigna, acredita-se na busca de ações, contemplando o controle laboratorial, com métodos padronizados e oficiais. 


\section{REFERÊNCIAS}

1. Agência Nacional de Vigilância Sanitária - Anvisa. Resolução RDC № 259, de 20 de setembro de 2002. Aprova o regulamento técnico para rotulagem de alimentos embalados. Diário Oficial União. 23 set 2002.

2. Agência Nacional de Vigilância Sanitária - Anvisa. Resolução RDC № 360, de 23 de dezembro de 2003. Aprova regulamento técnico sobre rotulagem nutricional de alimentos embalados, tornando obrigatória a rotulagem nutricional. Diário Oficial União. 26 dez 2003.

3. Brasil. Lei $n^{\circ} 6.437$, de 20 de agosto de 1977 . Configura infrações à legislação sanitária federal, estabelece as sanções respectivas, e dá outras providências. Diário Oficial União. 24 ago 1977.

4. Monteiro RA, Coutinho JG, Recine E. Consulta aos rótulos de alimentos e bebidas por frequentadores de supermercados em Brasília, Brasil. Rev Panam Salud Publica. 2005;18(3):172-7. https://doi.org/10.1590/S1020-49892005000800004

5. Marchi PGF, Rezende-Lago NCM, Pachemshy JAS. Hábito de leitura dos rótulos de alimentos lácteos por consumidores de supermercados em Sinop, MT. Rev Eletr Univar. 2011;2(6):189-95.

6. Brasil. Lei ${ }^{\circ} 8.078$, de 11 de setembro de 1990. Dispõe sobre a proteção do consumidor e dá outras providências. Diário Oficial União. 12 set 1990.

7. Cassemiro IA, Colauto NB, Linde GA. Rotulagem nutricional: quem lê e por quê?. Arq Ciênc Saúde Unipar. 2006;10(1):9-16.

8. Machado SS, Santos FO, Albinati FL, Santos LPR. Comportamento dos consumidores com relação à leitura de rótulo de produtos alimentícios. Alim Nutr Araraquara. 2006;1(17):97-103.

9. Marins BR, Jacob SC. Avaliação do hábito de leitura e da compreensão da rotulagem por consumidores de Niterói, RJ. Vigil Sanit Debate. 2015;3(3):122-9. https://doi.org/10.3395/2317-269x.00203

10. Rodrigues HF, Silva LFM, Ferreira KS, Nogueira FS. Avaliação de rotulagem nutricional, composição centesimal e teores de sódio e potássio em batatas-palha. Rev Inst Adolfo Lutz. 2010;69(3):423-7.

11. Anton AA, De Francisco A, Haas P. Análise físico-química de pães da cidade de Florianópolis e a situação dos alimentos integrais no Brasil. Alim Nutr Araraquara. 2006;17(4):381-6.

12. Lobanco CM, Vedovato GM, Cano CB, Bastos DHM. Fidedignidade de rótulos de alimentos comercializados no município de São Paulo, SP. Rev Saúde Pública. 2009;43(3):499-505. http://doi.org/10.1590/S0034-89102009005000020

13. Silva AM, Dutra MBL. Avaliação de conformidade da rotulagem nutricional de preparados instantâneos tipo “sopão”. Alim Nutr Araraquara. 2012;1(23):51-4.

14. Interscience Informação e Tecnologia Aplicada. Como atrair o consumidor infantil, atender expectativas dos pais e ainda, ampliar as vendas. São Paulo: Interscience
Informação e Tecnologia Aplicada; 2003[acesso 12 jun 2012]. Disponível em: http://criancaeconsumo.org.br/ wp-content/uploads/2014/02/Doc-09-Interscience.pdf

15. Ferreira JSG, Silva Y, Moraes OMG, Tancredi RP. Marketing de alimentos industrializados destinados ao público infantil na perspectiva da rotulagem. Vigil Sanit Debate. 2015;3(2):75-84. https://doi.org/10.3395/2317-269x.00293

16. Instituto Brasileiro de Geografia e Estatística - IBGE. Pesquisa de orçamentos familiares 2008-2009: antropometria, estado nutricional de crianças, adolescentes e adultos no Brasil. Rio de Janeiro: Instituto Brasileiro de Geografia e Estatística; 2010.

17. Instituto Adolfo Lutz. Métodos físico-químicos para análise de alimentos: normas analíticas do Instituto Adolfo Lutz. 4a ed. São Paulo: Instituto Adolfo Lutz; 2008.

18. AOAC International. Official methods of analysis of the Association of Official Analytical Chemists. 20th ed. Rockville: AOAC International; 2016

19. Pereira J, Silva RPG, Nery FC, Vilela ER. Comparação entre a composição química determinada e a declarada na embalagem de diferentes marcas de pão de queijo. Ciênc Agrotec Lavras. 2005;29(3):623-8. https://doi.org/10.1590/S1413-70542005000300017

20. Lima EE, Abreu TR, Silva MK, Parisenti J. Comparação entre os conteúdos de gorduras analisados experimentalmente e os reportados nos rótulos de empanadas comercializadas em cantinas escolares de Florianópolis, Santa Catarina. Demetra. 2016;11(1):121-34 https://doi.org/10.12957/demetra.2016.16167

21. Mello AV, Cassimiro TAS, Pospischek VS, Villarim WLF, Pereira IRO, Abreu ES. Avaliação da composição centesimal e da rotulagem de barras de cereais. e-Sci. 2012;2(5):41-8.

22. Pederzoli BA, Lourenço, AA, Botelho FT. Análise laboratorial de bolos destinados para público infantil e comparação com as informações nutricionais contidas nos rótulos e adequação à legislação. Rev Inst Adolfo Lutz. 2014;73(4):344-50. https://doi.org/10.18241/0073-98552014731627

23. Elliot P, Walker LL, Little MP, Blair-West JR, Shade Re, Lee DR et al. Change in salt intake affects blood pressure of chimpanzees: implications for human populations. Circulation. 2007;116(14):1563-8. https://doi.org/10.1161/CIRCULATIONAHA.106.675579

24. Geleinjse JM, Hofman A, Witteman JCM, Hazebroek AA, Valkenburg HA, Grovvee DE. Longterm effects of neonatal sodium restriction on blood pressure. Hypertension. 1997;29(4):913-7. https://doi.org/10.1161/01.HYP.29.4.913

25. Hofman A, Hazebroek AA, Valkenburg HA. $A$ randomized trial of sodium intake and blood pressure in newborn infants. JAMA. 1983;250(3):370-3. https://doi.org/1010.1001/jama.1983.03340030030023

26. Ministério da Saúde (BR). Guia alimentar para a população brasileira. 2a ed. Brasília, DF: Ministério da Saúde; 2015. 
27. Ministério da Saúde (BR). Termo de Compromisso $\mathrm{N}^{\circ}$ 004/2011, de 7 de abril de 2011. Termo de compromisso que firmam entre si a União, por intermédio do Ministério da Saúde, e a Associações Brasileiras das Indústrias de Alimentação (ABIA), a Indústrias Brasileira das Massas Alimentícias (ABIMA), a Associação Brasileira da Indústria de Trigo (ABITRIGO) e a Associação Brasileira da Indústria de Panificação e Confeitaria (ABIP), com a finalidade de estabelecer metas nacionais para a redução do teor de sódio em alimentos processados no Brasil. Diário Oficial União. 8 abr 2011.

28. Ministério da Saúde (BR). Secretaria de Vigilância em Saúde. Departamento de Análise de Situações de Saúde. Plano nacional de redução de consumo de sal. Brasília: DF: Ministério da Saúde; 2011.

29. Nilson EAF, Jaime PC, Resende DO. Iniciativas desenvolvidas no Brasil para redução do teor de sódio em alimentos processados. Rev Panam Salud Publica. 2012;32(4):287-92. https://doi.org/10.1590/S1020-49892012001000007

30. Dias GLE, Moraes OMG, Camara AO. Determinação quantitativa da concentração de sódio em pães tipo bisnaguinha comercializados na cidade do Rio de Janeiro. Vigil Sanit Debate. 2015;3(2):48-55. https://doi.org/10.3395/2317-269x.00441
31. Martins APB, Andrade GC, Bandoni DH. Avaliação do monitoramento do teor de sódio em alimentos: uma análise comparativa com as metas de redução voluntárias no Brasil. Vigil Sanit Debate. 2015;3(2):56-64. https://doi.org/10.3395/2317-269x.00323

32. Sauerbronn ALA. Análise laboratorial da composição de alimentos processados como contribuição ao estudo da rotulagem nutricional obrigatória de alimentos e bebidas embalados no Brasil [dissertação]. Rio de Janeiro: Instituto Nacional de Controle de Qualidade em Saúde; 2003.

33. Jesus CC, Rocha RCF, Laboissière LHES, Pereira AJG. Avaliação da composição média determinada e da apresentada na embalagem para diferentes marcas de pão de queijo. In: Anais do 180 Congresso Brasileiro de Ciência e Tecnologia de Alimentos; 2002, Porto Alegre. Porto Alegre: SBCTA; 1990.

34. Fregonesi BM, Yokosawa CE, Okada IA, Massafera G, Braga Costa TM, Prado SPT. Polpa de açaí congelada: características nutricionais, físico-químicas, microscópicas e avaliação da rotulagem. Rev Inst Adolfo Lutz. 2010;69(3):387-95.

35. BRASIL. Decreto № 5.903, de 20 de setembro de 2006. Regulamenta a Lei $\mathrm{N}^{\circ} 10.962$, de 11 de outubro de 2004 , e a Lei $N^{\circ} 8.078$, de 11 de setembro de 1990. Diário Oficial União. 21 set 2006.

Conflito de Interesse

Os autores informam não haver qualquer potencial conflito de interesse com pares e instituições, políticos ou financeiros deste estudo.

Esta publicação está sob a licença Creative Commons Atribuição 3.0 não Adaptada.

Para ver uma cópia desta licença, visite http://creativecommons.org/licenses/by/3.0/deed.pt_BR. 